\title{
UNIVERSUM
}

\section{TRADUCIR EL CARIBE ANGLÓFONO EN LA REVISTA CASA DE LAS AMÉRICAS: LA REARTICULACIÓN DEL DISCURSO NUESTROAMERICANO}

\author{
Translating the Anglophone Caribbean in Cuba's Casa de las Américas: The Re- \\ Articulation of Discourse on Our America
}

\author{
Thomas Rother (iD) \\ ${ }^{1}$ Universidad de Chile. Facultad de Filosofía y Humanidades, Nuñoa, RM, Chile.
}

thomasrothe@ug.uchile.cl

\section{RESUMEN}

Aunque Casa de las Américas es reconocida como una de las revistas culturales más influyentes en el campo intelectual latinoamericano, su papel de promotor de la traducción literaria ha sido poco estudiado. Este artículo se centra en cómo la revista traduce y difunde conocimiento sobre la producción literaria del Caribe anglófono, una de las zonas lingüísticas de la región que tuvo mayor presencia en la revista durante la década de los 7o. Se plantea que, con este acercamiento, la revista articula una nueva dimensión de su discurso nuestroamericano, que busca establecer unidad entre los países subdesarrollados de América en pro de una afirmación identitaria que valora lo propio y resiste el colonialismo cultural. La gran cantidad de traducciones que puso en circulación la revista Casa en esta época constituye un hito en la historia de la traducción del Caribe y América Latina y su análisis en este artículo busca diseccionar los intercambios, enfocándose en los puntos de encuentro y desencuentro.

PALABRAS CLAVE: Casa de las Américas; traducción; caribe anglófono; revistas culturales; discurso nuestroamericano.

\section{ABSTRACT}

While Casa de las Américas is recognized as one of the most influential cultural magazines in the Latin American intellectual field, its role in promoting literary translation has received little attention. This article focuses on how the magazine has translated and spread knowledge of literature written in the Anglophone Caribbean, one of the areas with the largest presence in the magazine during the r97os. This interchange articulated a new dimension of the magazine's discourse regarding Our America, which sought to establish unity among underdeveloped countries throughout the Americas as a means to assert regional and national identities amidst the onslaught of cultural imperialism. The enormous number of translations that Casa published in this period constitutes a milestone in the history of translation in the Caribbean and Latin America. The analysis in this article looks to dissect the exchanges, focusing on intersections of similarities and difference.

KEYWORDS: Casa de las Américas: translation; anglophone Caribbean; cultural magazines; discourse on our America.

Fecha de Recepción $2020-03-02$
Fecha de Evaluación 2020-09-25
Fecha de Aceptación

$2020-I I-20$ 


\section{INTRODUCCIÓN}

Dentro del panorama de revistas culturales latinoamericanas y caribeñas, Casa de las Américas se destaca por su duración e influencia en el campo cultural. Si bien la revista cuenta con una contundente bibliografía crítica que se centra en su papel catalizador de la intelectualidad latinoamericana de los años 6o (Weiss 1973; Lie 1996; Fornet 200I; Campuzano 200I; Gilman 2003, 2018; Morejón Arnaiz 2017), pocos estudios han abordado su trabajo de traducción, que atraviesa el proyecto editorial y constituye un rico e inmenso corpus de poesía, cuentos, fragmentos de novelas y ensayos vertidos desde lenguas tan diversas como el ruso y el creole haitiano. Este artículo se centra específicamente en cómo la revista Casa tradujo la producción literaria del Caribe anglófono para un público latinoamericano hispanohablante, labor que adquirió protagonismo a partir de los 70 y expandió las redes de la familia latinoamericana de intelectuales de izquierda. En medio de una crisis de legitimidad provocada por el caso Padilla y el llamado quinquenio gris, las políticas culturales cubanas buscaron fortalecer alianzas en los países vecinos del Caribe anglófono, que habían recién empezado su proceso de independencia en la década del 6o. En este escenario, la traducción de autores de esta subregión caribeña permitió la rearticulación del discurso nuestroamericano, desde una retórica de integración hacia un esfuerzo concreto de intercambio intelectual que atravesará las lenguas del continente, rompiendo antiguas barreras de comunicación instaladas por el colonialismo europeo y sus secuelas. María Constanza Guzmán ha leído esta política de traducción intracaribeña en la revista como "una praxis descolonial, en la medida en que el tejido narrativo se proyecta hacia sí mismo como parte de un proyecto intelectual americano, en construcción dinámica y en proceso, y a la vez irradia dicho proyecto hacia el continente" (I79). El análisis desarrollado en este artículo se centra en los intercambios realizados con una zona lingüistica del Caribe en específico para revelar los puntos tanto de encuentro como de desencuentro al nivel estético, ideológico y cultural.

\section{PROYECTO EDITORIAL: CUBA EN NUESTRA AMÉRICA}

Casi en paralelo con el proceso revolucionario, la revista Casa de las Américas ha circulado desde junio de 1960, con una frecuencia bimestral que, solo a partir de los 9o, se vio obligada a bajar a trimestral debido al aprieto económico durante el Periodo Especial. Durante su apogeo de impresión en los años 70, llegó a tener un tiraje de 15.000 ejemplares por número con un promedio de 200 páginas, dando cuenta de una de las más difundidas y contundentes publicaciones culturales en el continente. Pese a contar con auspicio estatal, la revista Casa nunca fue un órgano oficial de 
las políticas gubernamentales, manteniendo cierto grado de autonomía que contribuía a su legitimidad en el campo cultural latinoamericano. Es más, los editores y colaboradores durante los primeros años se formaron en espacios como Ciclón y Lunes de Revolución, la publicación dirigida por Guillermo Cabrera Infante que terminó en 196r después del llamado "caso PM", primera polémica del periodo revolucionario (Campuzano 37). Así, nombres como José Rodríguez Feo, Virgilio Piñera, Calvert Casey, Óscar Hurtado, Edmundo Desnoes, Néstor Almendros, entre otros, predominan en las páginas de los primeros años de la revista Casa. Como señala Gilman, el equipo internacional de colabores en esta época también problematiza la caracterización de la revista como una representación única y oficial de la Revolución: Ángel Rama, Julio Cortázar, Manuel Galich, Ezequiel Martínez Estrada, Miguel Ángel Asturias no solo publicaron textos de su autoría, sino que colaboraron en la coordinación de números especiales o en la redacción de los editoriales ${ }^{1}$ ("Los primeros años..." I37). Sin embargo, el funcionamiento de la revista es inseparable de la institución homónima y el trabajo coordinado de las dos transformó el espacio de la Casa de las Américas en un núcleo articulador de la intelectualidad latinoamericana durante los 6o: la organización de congresos internacionales permitían un punto de contacto físico entre intelectuales, escritores y artistas del continente; el Premio Literario Casa de las Américas, que al principio se llamaba "Concurso literario hispanoamericano", conformaba jurados internacionales y premiaba obras de diferentes escritores latinoamericanos con una frecuencia anual; y la revista difundía una gran cantidad de material literario y crítico latinoamericano, muchas veces resultado de las actividades organizadas por la Casa ${ }^{2}$.

El discurso nuestroamericano alentado por la dirección de Fernández Retamar a partir de la segunda mitad de la década del 6o, ofrece un marco conceptual para comprender la heterogeneidad cultural y política del continente. Esta categoría se entiende como un posicionamiento identitario que busca valorar elementos culturales propios frente a la hegemonía euro-occidental y establecer vínculos entre los países subdesarrollados del continente. En el número 30 de 1965 , el primero en que aparece Fernández Retamar como Director, su editorial manifiesta la intención de adoptar esta trinchera discursiva como línea editorial:

Esperamos en entregas posteriores, de acuerdo con sus principios y con la valiosa tarea ya cumplida por la Casa de las Américas, las que del río Bravo a la Patagonia son una, decía Martí, «en el origen, en la esperanza y en el peligro»: los países que ahora llaman subdesarrollados, llenos

\footnotetext{
${ }^{1}$ Según Gilman, Rama redactó la nota editorial del número 2 y coordinó el emblemático número 26 de octubrenoviembre, 1964, que tuvo una influencia mayor en la articulación de la nueva novela latinoamericana como fenómeno literario ("Los primeros años...", I37).

2 Todas estas actividades siguen siendo realizadas por la Casa en la actualidad, aunque su papel articulador de la intelectualidad latinoamericana ha perdido centralidad.
} 
de conflictos y de porvenir, a los que estamos entranablemente unidos, y de cuya comunidad de creación artística, intelectual, humana, como de sus problemas más reales no hay fuerza capaz de separarnos. Es tarea creciente de esta revista contribuir a ratificar esa identificación. (cit. en Fernández Retamar "El Caribe..." 179-180)

Al igual que la definición aportada por Martí en I89r para establecer una distinción con la otra América anglosajona, el concepto geo-cultural que Fernández Retamar reinyecta al debate comprende todo el continente al sur de EE. UU. y explicita la pertenencia tanto de los pueblos indígenas como de todo el Caribe, independiente de la lengua. Así, en su ensayo "Nuestra América y Occidente", publicado en la revista Casa en 1976, Fernández Retamar define el término de la siguiente manera: "La 'América Latina' (más allá de lo que en un principio se quiso que esta expresión significara, es decir, tomada ahora como sinónimo de 'nuestra américa') incluye no solo pueblos de relativa filiación latina, sino también otros, como los de las Antillas de lengua inglesa u holandesa, más bien alejados de tal filiación; y, por supuesto, los grandes enclaves indígenas" (n.. $98,36)$.

A lo largo de estas dos décadas, el vínculo entre el intelectual y su compromiso político estuvo fuertemente cuestionado en el campo cultural latinoamericano, apoyado por voces críticas como Carlos Fuentes, Pablo Neruda, Mario Vargas Llosa y cubanos exiliados en Europa o EE. UU., como Cabrera Infante y Severo Sarduy, colaboradores con el proyecto revolucionario durante sus primeros años. El caso Padilla, que cierra el ciclo de los efervescentes años 6o, sin duda termina de trizar las lealtades de la intelectualidad latinoamericana, abriendo la nueva década con dos flancos ideológicos que disputan el liderazgo de la literatura y el pensamiento del continente, debates que se explayan en la revista Casa y su contrincante Mundo Nuevo. En este delicado escenario, las relaciones entre la Casa y la intelectualidad anglocaribeña surgen de manera muy orgánica, encontrando en las islas vecinas un prototipo de escritor que cumplía con las exigencias de un intelectual público, comprometido con la lucha anticolonial y el desarrollo de la cultura nacional. Autores como George Lamming, Kamau Brathwaite, Jan Carew, Andrew Salkey, y críticos como Keith Ellis y Rex Nettleford encontraron afinidades políticas y culturales entre los pasillos de la Casa y entre las páginas de su revista. El quiebre con ciertos sectores de la intelectualidad latinoamericana permitió que la Casa buscara reforzar la legitimidad de su discurso en el resto del Caribe, espacio geográfico y cultural al cual siempre ha pertenecido, pero que conocía poco, debido a las diferencias lingüísticas y separaciones coloniales. 


\section{POLITTICAS DE TRADUCCIÓN EN CASA: APERTURA HACIA EL CARIBE}

Si bien la revista no gira en torno a la traducción, esta constituye una práctica fundamental del proyecto editorial. Entre 1960 y 1989, Casa publicó cerca de 400 textos traducidos, de los cuales predominan los géneros de ensayo y poesía, pero también hay considerables muestras de narrativa y teatro $^{3}$. No existe otra revista caribeña que haya puesto en marcha un aparato de traducción de características similares, estableciéndose como un hito para la historia de la traducción de la región. La gran cantidad de traducciones que publica Casa, junto con la tendencia a visibilizar a los traductores, sean profesionales, aficionados de lenguas extranjeras o escritores conocidos, indica que la revista concibe la traducción como un ejercicio literario e intelectual legítimo y valioso. Mientras la difusión del trabajo de autores extranjeros se alinea con los objetivos internacionalistas de la Revolución ${ }^{4}$, el discurso que la revista desarrolla sobre la traducción, su presencia en debates y paratextos, adopta diferentes características a lo largo de estos años, marcadas en gran parte por las circunstancias políticas de la isla.

Durante la primera etapa de la revista, cuando la dirección oscila entre Antón Arrufat, Fausto Masó y Pablo Armando Fernández, los textos traducidos se limitan casi exclusivamente al género de ensayo, con una proporción dominante de autores estadounidenses. Cuando Fernández Retamar asume como director en 1965 , sucede un notable cambio en la procedencia de los autores traducidos: disminuyen los estadounidenses, cediendo paso a los europeos, pero también entran más caribeños, en especial del área francófona, entre ellos René Depestre5 , Aimé Césaire y Gérard Pierre-Charles. Mientras se traduce más poesía, el género dominante de los textos traducidos en esta etapa sigue siendo el ensayo. Otra tendencia muy marcada en la segunda mitad de los 6o es el aumento considerable de traducción de autores marxistas y de la Unión Soviética, paralelo al

\footnotetext{
3 Tomo estas fechas por el periodo que he estudiado en mi tesis doctoral, titulada "Un prisma bajo el sol: traducción en revistas literarias del Caribe hispanohablante y el Caribe anglófono", realizada en el Doctorado en Literatura de la Universidad de Chile. La cifra es aproximada porque excluye muchos textos de carácter informativo, declaraciones hechas por organismos y la gran cantidad de mensajes que llegaron a la Casa desde el extranjero y que terminaron siendo publicados en la revista. Asimismo, el conteo excluye la publicación de ponencias presentadas en español, considerando que generalmente corresponden a un proceso de traducción previo en el cual no se identifica el traductor. Para los textos de René Depestre, Margaret Randall y Keith Ellis en los que no señalan el traductor, es difícil determinar si son traducciones o si los escribieron directamente en español, debido a que todos eran hispanohablantes. Cuando no hay traductor señalado en estos casos, asumo que los autores escribieron los textos en español y no los he considerado dentro del conteo de traducciones, aunque, claramente representa un proceso de traducción que merece ser estudiado.

${ }^{4}$ Como señala Carlos Rafael Rodríguez, la política exterior de Cuba se basa en los principios marxistas-leninistas que ven la lucha de clases en un nivel local e internacional, por lo que una de sus premisas estratégicas es el internacionalismo (III3).

5 Aunque Depestre, exiliado de Haití durante el régimen de Francois "Papa Doc" Duvalier, tomó residencia en Cuba en I959 e inauguró su presencia en Casa desde el número 2, con la publicación del poema "Caídos en el campo de la claridad" y un artículo crítico sobre las elecciones presidenciales en los EE.UU., ninguno con indicación de traductor.
} 
acercamiento político de Cuba con el campo soviético. Si bien los autores del Caribe anglófono empiezan a ser traducidos a partir de 1968 , su participación en la revista aumenta a lo largo de los 70, siendo uno de los grupos de autores extranjeros con mayor presencia. A partir de 1973, se comienza a señalar el idioma del que se traduce, antepuesto al nombre del traductor, que expone la gran diversidad de lenguas desde las cuales los colaboradores de la revista escriben: inglés, francés, alemán, checo, catalán, italiano, portugués, húngaro, eslovaco, entre otros. En la década de los 7o también se suele citar la traducción como una "versión española", indicando una mayor reflexión sobre el proceso de traducir y el texto final no necesariamente como un reflejo fiel del original.

Autores como C.L.R. James, George Lamming y Andrew Salkey constituyen algunos de los primeros contactos de la Casa con la intelectualidad anglocaribeña. En el número 48 de I968, James publica un ensayo sobre el movimiento del Poder Negro en los EE.UU. y, en particular, uno de sus líderes, Stokely Carmichael, estadounidense de origen trinitario, a quien compara con George Padmore, Marcus Garvey, Césaire y Frantz Fanon. Un año después, en el número 56, se publican por primera vez las palabras de Lamming a través de su texto "Actitudes de literatura antillana con respecto a África", una intervención leída en el Simposio sobre la Influencia Africana en las Literaturas Antillanas, realizada en la Universidad Central de las Villas en diciembre de ig68. Sin indicar el traductor, el texto sitúa la base de la poesía anglocaribeña como producto de la condición colonial, utilizando ejemplos de Vera Bell, Derek Walcott y E.M. Roach. Si bien Salkey mantuvo correspondencia con trabajadores de la Casa desde su viaje a Cuba para participar en el Congreso Cultural de La Habana en 1968, no fue hasta 1974, en el número 86, que apareció su poesía en la revista. Se tratan de dos poemas, "Un pueblo unido" y "Colaboradores cautivos", escritos a partir del golpe de estado cívico-militar que se dio en Chile el in de septiembre de i973. Salkey, quien conoció a Joan Jara ${ }^{6}$ en Inglaterra, tomó un vivo interés en la lucha chilena contra la dictadura y la conservación de la memoria de los ejecutados políticos y detenidos desaparecidos. Los dos poemas publicados en la revista en 1974 integran parte de su libro In the Hills Where Her Dreams Live, que mira la tragedia chilena desde una perspectiva caribeña, abriendo el poemario con una comparación metafórica entre la pérdida personal de una mujer jamaicana y la pérdida nacional de la Unidad Popular. Los poemas son traducidos por A.Z., seudónimo para las traducciones colectivas que, en este caso específico, se atribuyen a Blanca Acosta, Adelaida de Juan y Fernández Retamar (Fernández Retamar a Salkey, i nov. 1974). Estos poemas constituyen los primeros poemas de un autor anglocaribeño publicados en la revista y articuladores de las nuevas dimensiones que empezó a tomar el discurso nuestroamericano de Casa en esta época.

\footnotetext{
${ }^{6}$ Viuda de Víctor Jara, cantautor chileno ejecutado en septiembre de 1973.
} 
Alrededor del mismo tiempo, surgen esfuerzos desde la crítica cubana y de extranjeros residentes en Cuba que empiezan a ilustrar una inmensa y compleja producción literaria del Caribe anglófono. Jacqueline Kaye, crítica literaria inglesa que trabajaba en la Facultad de Lenguas Extranjeras de la Universidad de La Habana, es la autora de dos textos fundamentales que aparecen en Casa en esta época: "La historia en la novela antillana de lengua inglesa", traducido por Luisa María Jané (n.. 67, I97I); y "Literatura y subdesarrollo y el subdesarrollo de la crítica literaria", (n.․․ 74, 1972), sin identificación de un traductor. El primero ofrece un análisis detallado de cómo los novelistas de la subregión han lidiado con los problemas de la historia caribeña, en particular el peso de la esclavitud, el prolongado colonialismo y las obligaciones de exiliarse. El segundo ensayo cuestiona varios supuestos comunes en la crítica anglosajona, como la creencia de que la literatura anglocaribeña es un fenómeno del siglo XX o la necesidad de valer la literatura a partir de una noción de tradición. Uno de los ensayos más importantes sobre la literatura anglocaribeña se publica en el número 86 de 1974: "Introducción a la narrativa antillana de lengua inglesa", escrito por Blanca Acosta, Samuel Goldberg e Ileana Sanz. Pese al título alusivo a la narrativa, el ensayo brinda un panorama bastante completo del desarrollo y la producción contemporánea de la literatura en el Caribe anglófono, deteniéndose incluso en la función de las revistas literarias como plataformas fundamentales para la difusión de esta literatura en ciernes: así, aborda el trío clásico de The Beacon, BIM y Kyk-Over-Al y algunas revistas contemporáneas, entre ellas Savacou, editada en los 70 por Kamau Brathwaite y Kenneth Ramchand en Jamaica. La publicación del texto de Acosta, Goldberg y Sanz se enmarca en el establecimiento de relaciones diplomáticas entre Cuba y los gobiernos de Barbados, Jamaica, Guyana y Trinidad y Tobago, logrado el in de diciembre de 1972. La cooperación entre el gobierno de Fidel Castro y los primeros ministros de estos países recientemente independizados se basó en objetivos ideológicos afines: terminar con la acumulación histórica del colonialismo en el Caribe y lograr la liberación y soberanía nacional.

\section{EL NÚMERO 9I: "LAS ANTILLAS DE LENGUA INGLESA"}

El año I975 marca un verdadero hito en los intercambios entre Cuba y el Caribe anglófono con la publicación del número 9r de Casa, "Las Antillas de lengua inglesa", concebido en conjunto con un número paralelo de la revista jamaicana Caribbean Quarterly (CQ), dedicado a la literatura y cultura cubanas (vol. 2I, n.ำ I/2, I975). En su análisis comparativo de estos números especiales, Luz Rodríguez-Carranza y Nadia Lie argumentan que, pese a la intención de formular un punto de

\footnotetext{
7 Incluido después como la introducción de la emblemática antología bilingüe Cuentos del Caribe (i977).
} 
encuentro mediante una retórica de identificación y cooperación entre ambos países, las distancias de percepción espacio-temporal inhiben la proyección de un discurso realmente común (I26). En términos traductológicos, también existen discrepancias en el contenido de cada número: mientras CQ traduce poemas de Nicolás Guillén y Fernández Retamar, una entrevista con Carpentier y una carta rescatada de Antonio Maceo, completando el resto del contenido con artículos de autores jamaicanos, Casa articula un enorme esfuerzo de traducir al español más de cuarenta escritores y críticos anglocaribeños, procedentes de Jamaica, Trinidad y Tobago, Barbados, Santa Lucía, Guyana, Dominica y San Vicente, la mayoría traducidos por primera vez al español. Por otro lado, mientras el intercambio para $C Q$ no significa una apertura perdurable hacia la intelectualidad y producción cultural latinoamericana, el número de Casa no solo se presenta como una novedad editorial al público hispanohablante, sino que también como un esfuerzo por crear redes intelectuales y reforzar la lectura y el prestigio de la revista Casa en el área anglófona de la región. Esto se ve en la inédita decisión de publicar en formato bilingüe tanto el editorial de Fernández Retamar como un discurso de Fidel Castro ${ }^{8}$, además de las bases del Premio Literario 1976, año en que abre el concurso a autores caribeños de lengua inglesa en la categoría de ficción?

En el editorial, Fernández Retamar delinea semejanzas religiosas, culturales y, sobre todo, históricas entre las distintas zonas lingüísticas del Caribe. Este esfuerzo discursivo busca integrar nuevos horizontes críticos y literarios del Caribe al campo cultural cubano al mismo tiempo que afirma la pertenencia caribeña de Cuba. Pero también hace un llamado explícito a considerar el Caribe anglófono como parte de América Latina, aunque reconoce que la mayoría de los anglocaribeños no se sienten latinoamericanos y optan por identificarse como West Indian. Sin ir más allá de señalar el error histórico de tal denominación, Fernández Retamar plantea el gentilicio como un problema de traducción y aprovecha para señalar algunas decisiones que se tomaron a lo largo del número: West Indies se traduce como Antillas y, en muchos casos, como Antillas de lengua inglesa, sin que el idioma estuviese indicado en el texto original. Esta denominación, que, si bien no incluye las variaciones de creol surgidas en el área anglófona, establece una norma terminológica consistente para los colaboradores de la revista hasta la segunda mitad de la década de los 8o, cuando se empieza a usar con más frecuencia el término Caribe anglófono ${ }^{10}$.

\footnotetext{
${ }^{8}$ Pronunciado en un acto de recepción del Primer Ministro de Guyana, Forbes Burnham, en la ciudad de Cienfuegos el 9 de abril de 1975 .

9 Solo el editorial señala que la traducción al inglés es de Margaret Randall. El número de CQ también publica las bases del Premio Literario de Casa de las Américas 1976 en inglés.

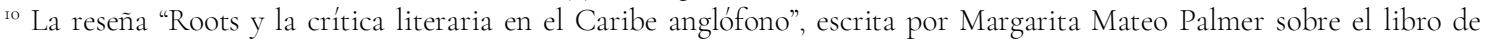
ensayos de Kamau Brathwaite, publicada en el número 164 de 1986, es el primer texto en la revista que utiliza el término Caribe anglófono en vez de Antillas de lengua inglesa. Desde entonces, el término se ha integrado al vocabulario de
} 
Aparte de los textos bilingües, otra característica novedosa de este número es el gran protagonismo autorial del Caribe anglófono: las secciones "Hechos/ideas", "Documentos" y "Letras" incluyen exclusivamente textos de escritores e intelectuales anglocaribeños, mientras "Notas", "Artes plásticas" y "Libros" publican textos de cubanos y un estadounidense residente en Cuba, cada sección con un solo texto. Sin duda la sección más contundente, "Letras", da a conocer más de cien páginas del trabajo literario de autores anglocaribeños, siguiendo un orden cronológico de su producción. Aparte de Claude McKay, todos son poetas y narradores contemporáneos, incluyendo Frank Collymore (Barbados), Jean Rhys (Dominica), Philip M. Sherlock (Jamaica), Roger Mais (Jamaica), Edgar Mittelholzer (Guyana), V.S. Reid (Jamaica), Eric Roach (Tobago), A.J. Seymour (Guyana), Louise Bennett (Jamaica), John Figueroa (Jamaica), Wilson Harris (Guyana), George Campbell (Jamaica), A.N. Forde (Barbados), Samuel Selvon (Trinidad), Neville Dawes (Jamaica), Owen Campbell (San Vicente), John Hearne (Jamaica), Martin Carter (Guyana), John Wickham (Barbados), George Lamming (Barbados), Bruce St. John (Barbados), John La Rose (Trinidad), Andrew Salkey (Jamaica), Derek Walcott (Santa Lucía), Kamau Brathwaite (Barbados), Austin Clarke (Barbados), Ian MacDonald (Trinidad), Timothy Callender (Barbados), Mervyn Morris (Jamaica), Dennis Scott (Jamaica) y Anthony McNeill (Jamaica). Pese a reunir un amplio conjunto de autores de diversos territorios de la subregión, Fernández Retamar, en su editorial, es enfático en señalar que la entrega no tiene "pretensión antológica, ya que carecemos de información para ello, sino la voluntad de ofrecer unas cuantas muestras del alto nivel alcanzado por esa literatura" (n. 9 9I, 5). Reconoce de esta manera la falta de comunicación y conocimiento de la producción literaria e intelectual anglocaribeña.

Los traductores que se encargaron de verter al español los textos incluidos en este dossier tienden a ser escritores cubanos ya consagrados, incluyendo a Eliseo Diego (Collymore, Figueroa), Antonio Benítez Rojo (Rhys, La Rose), Manuel Moreno Fraginals (Reid), David Chericián (Bennett, Brathwaite, Scott), Roberto Fernández Retamar (George Campbell, Owen Campbell, Carter, Walcott, MacDonald), Luis Suardíaz (Forde, Morris), Cos Causse (Dawes), Samuel Feijóo (Salkey) y Ambrosio Fornet (Callender). Solo hay un traductor extranjero: Mario Benedetti, miembro permanente del comité de colaboración, quien tradujo uno de los poemas más conocidos de McKay, "Si debemos morir". Este grupo representa lo que Patricia Willson ha llamado traductores-escritores, cuya autoría impuesta sobre el texto traducido influye en la lectura de autores generalmente desconectados o desconocidos dentro del campo cultural receptor (36). Por

muchos escritores, críticos e intelectuales vinculados a la Casa de las Américas, como se muestra en los trabajos de Fornet (24) y Campuzano (55). 
otro lado, hay una marcada presencia de traductoras profesionales o en formación, como Esther Muñiz, quien traduce un cuento de Wickham, Esther Pérez, quien aporta el fragmento de novela de Mittelholzer, y Blanca Acosta, quien ofrece un fragmento de The Palace of the Peacock de Harris, además de traducir un cuento de Clarke. Tanto Muñiz como Acosta tendrían un papel fundamental en el proyecto pancaribeño de la Casa: la primera tradujo la celebrada novela de Mais The Hills Were Joyful Together (1953) con el título Las montañas jubilosas (1978), y la segunda se hizo cargo - junto con Ileana Sanz y Samuel Goldberg - de la selección y traducción de Cuentos del Caribe (1977), la primera antología bilingüe de narrativa anglocaribeña en un país de habla hispana (Sanz 184). El dossier también publica traducciones colectivas, como en los casos de A.R. y Joaquín G. Santana, quienes vierten al español un poema de Sherlock y otro de Roach ${ }^{\text {II }}$, y Trinidad Mendoza y Francisco Garzón Céspedes, quienes traducen los poemas de St. John y McNeill. Los traductores menos conocidos son la excepción, como Juan Jiménez, quien traduce un cuento de Lamming; Roberto Álvarez Pérez, quien traduce un fragmento de la última novela de Mais, Black Lightning (Relámpago negro); y Ramón Font, quien traduce un fragmento de la novela de Williams, Other Leopards (Otros leopardos). Los únicos textos que no dan crédito de la traducción son los cuentos de Selvon y Hearne, aunque el primero incluye una nota anónima a pie de página, señalado como "N. del T."

Al fijarse en los traductores, emerge una estructura divisoria entre el género literario y el perfil del traductor: mientras los escritores consagrados se hacen cargo de la poesía, los traductores profesionales, pocos conocidos o anónimos son responsables de la narrativa. Esto se puede explicar quizás por el mayor prestigio que la revista le atribuye a la poesía y las dificultades —o, como argumentan algunos, la imposibilidad (Jakobson I30-I3I) - de traducirla. Si bien las páginas de Casa promovieron el debate en torno a la nueva narrativa latinoamericana, no es menor que el director durante la mayor parte de su existencia fue un poeta, epíteto que prefirió ante el de intelectual o político ${ }^{\mathrm{I2}}$. Por otro lado, llama la atención que muchos de los traductores de este dossier no son los mismos que finalmente vierten al español algunas de las obras más importantes de la literatura anglocaribeña en las ediciones posteriores publicadas por el fondo editorial Casa. Es

\footnotetext{
" A.R. es un seudónimo utilizado por Adelaida de Juan y Roberto Fernández Retamar para indicar un trabajo de traducción en conjunto, similar al de A.Z., utilizado tanto en este número como anteriores. En la antología Poetas del Caribe anglófono, publicada por Ediciones Casa de las Américas en 20II, los mismos poemas de Sherlock y Roach aparecen traducidos por De Juan, Fernández Retamar y Santana (Tomo i, páginas 139 y 269). La invisibilidad de De Juan como traductora en la revista llama la atención, pero estas contribuciones anónimas sugieren que tuvo una influencia en las decisiones editoriales mucho más significativa de lo que aparenta.

${ }^{12}$ En entrevista con Goffredo Diana y John Beverly, Fernández Retamar comenta: "Así como no soy una personalidad política, según acabo de recordar, tampoco soy un filósofo: soy un poeta metido en camisa de once varas" (Un poeta metido... 93).
} 
el caso de las obras de Lamming, todas traducidas por María Teresa Ortega y no Jiménez; o Jean Rhys, de quien la edición cubana de El vasto mar de los sargazos se debe a Raquel Costa y no a Benítez Rojo. Esto ratifica el carácter inicial que representa el número en la divulgación de la literatura anglocaribeña en Cuba y en el resto de la América hispanohablante.

Los poemas y textos narrativos del dossier forman un conjunto muy variado de temas, entre ellas la personificación de la naturaleza, la insularidad psicológica, retratos de la vida campesina, los conflictos de clase y raza, aspectos de la historia regional y la diáspora. Dentro de esta heterogeneidad temática, aparecen dos líneas claras: el reconocimiento de la identidad cultural afrodescendiente y la afirmación nacional en clave anticolonial. En cuanto a la última, se manifiesta a través de poemas como "El mañana pertenece al pueblo" de A.J. Seymour y traducido por Moreno Fraginals. De verso directo, el poema celebra las clases obreras y visibiliza la situación colonial de explotación de recursos naturales y humanos. Otro poema en esta línea es "En la cuarta noche de una huelga de hambre" de Martin Carter, traducido por Fernández Retamar. Este texto autobiográfico y urgente forma parte de una serie de poemas que Carter escribió desde la cárcel en Guyana, donde cumplió una condena de seis meses en los años 5o, acusado por el gobierno colonial de incitar disidencia. Otros poemas de verso menos directo también caen bajo esta categoría anticolonial, como el de Kamau Brathwaite, "Oh sueños Oh destinos", traducido por Chericián, que relata el regreso a un Caribe intervenido por el imperialismo estadounidense y dominado por democracias corruptas; o "Velada literaria en Jamaica", de Mervyn Morris, traducido por Suardíaz, donde el hablante cuestiona la relevancia de la poesía británica.

Los textos que siguen una línea anticolonial y de afirmación nacional encajan fluidamente con el discurso político de Casa y, en este sentido, no es menor que Fernández Retamar traduce el poema de Carter, cuya poesía volvería a aparecer en la revista en el número I25 de ig8I, pero por la mano traductora de Chericián. No obstante, el director de la revista también traduce un poema de Walcott que sale de este marco: se trata de "Ruinas de una casa de vivienda", uno de los textos que la crítica frecuentemente utiliza para ejemplificar la relación ambigua de Walcott con la historia de la esclavitud y el colonialismo. Unas estrofas de la versión cubana al lado del original ilustran algunas decisiones tomadas en el proceso de traducción:

\footnotetext{
"Ruinas de una casa de vivienda"

Piedras tan solo, los dispersos trozos de esta /mansión

(Cuyas muchachas, semejantes a alevillas,

Se confunden con polvo de bujías),
}

"Ruins of a Great House"

Stones only, the disjecta membra of this Great /House,

Whose moth-like girls are mixed with candledust, Remain to file the lizard's dragonish claws. 
Quedan para afilar las garras dragonescas del /lagarto;

Las bocas de esos ángeles del porrón, /herrumbrosas;

El eje con la rueda del carruaje, enfangados bajo el /estiércol

Dejado por las reses.

[...]

Un verde césped, roto por pequeños muros de /piedra,

Descendía hasta el riachuelo, y recorriéndolo, pensé

En hombres como Hawkins, Walter Raleigh y /Drake,

Asesinos y poetas ancestrales, más confundidos

En la memoria ahora por cada crimen lacerante.

La verde edad del mundo entonces era una lima /pudriéndose,

Cuyo hedor se hizo el lema del galeón sepulcral.

La podredumbre queda con nosotros, los hombres /se han marchado.

Mas como la ceniza muerta es levantada por un /viento,

Que aviva la negreante pavesa de la mente,

Mis ojos incendió la cinérea prosa de Donne.

(Casa, no 9I, 1975, I64-165)
The mouths of those gate cherubs shriek with stain; Axle and coach wheel silted under the muck Of cattle droppings.

[...]

A green lawn, broken by low walls of stone, Dipped to the rivulet, and pacing, I thought next Of men like Hawkins, Walter Raleigh, Drake, Ancestral murderers and poets, more perplexed In memory now by every ulcerous crime.

The world's green age then was a rotting lime Whose stench became the charnel galleon's text The rot remains with us, the men are gone. But, as dead ash is lifted in a wind That fans the blackening ember of the mind, My eyes burned from the ashen prose of Donne. (Walcott 19-20)

El título de la versión de Retamar entrega una primera pista sobre las intenciones de ubicar el texto en la memoria de la esclavitud cubana. En vez de optar por la traducción literal de "gran casa", "casa de vivienda" alude a la terminología utilizada en textos literarios e historiográficos sobre las plantaciones azucareras en Cuba, presente en la obra de Anselmo Suárez y Romero en el siglo XIX y Fernando Ortiz en el siglo XX. Sacrifica así el sentido grandilocuente que existe en la demarcación de la gran casa o la casa grande en los países del Caribe anglófono y hasta en Brasil ${ }^{13}$ para traer el texto al público cubano y posiblemente generar resonancias más cercanas. Además, el primer verso deja claro que no se trata de cualquier casa, sino de una mansión. Por otra parte, y en diálogo con el original, existe una apuesta estética para recrear un lenguaje más arcaico, presente en decisiones como "mas" en vez de "pero", "Colonia cual nosotros" o de colocar el adjetivo frente al sustantivo, como en "La verde edad del mundo". Incluido en In a Green Night (I962), que recopila algunos de los poemas más jóvenes de Walcott, el poema original confirma que Fernández Retamar se guía por una lectura cercana del original, pero sin necesariamente buscar la equivalencia total. Lejos de ser un texto representativo de la poesía coloquial, el poema en inglés utiliza un amplio registro de lenguaje y citas intertextuales con la poesía inglesa (principalmente William Blake y John Donne). La ambigüedad que expresa Walcott en torno al pasado colonial en

\footnotetext{
${ }^{13}$ Ver Gilberto Freyre Casa-Grande y Senzala (1933).
} 
un momento histórico cuando el despertar nacional anglocaribeño reclamaba la posibilidad de reconocer la retención cultural africana en el Caribe $^{14}$, sale del marco común de la época y cuestiona las maneras de hacer memoria a través de la escritura poética. La traducción al español de Fernández Retamar recrea estos matices dentro de los parámetros culturales cubanos y, así, amplía el espectro de la poesía política que promueve la revista Casa.

En cuanto al reconocimiento de la identidad cultural afrodescendiente, los poemas y textos narrativos seleccionados en el dossier tienden a centrarse en aspectos históricos de la presencia africana en el Caribe más que en una reivindicación política contemporánea. Por lo tanto, en vez de introducir textos literarios que problematizan la histórica opresión del afrocaribeño y la estructuración racial configurada por las colonias inglesas, el dossier ofrece una serie de textos que dicen relación con el discurso transcultural cubano, corriendo el riesgo de invisibilizar las estructuras de poder y violencia inherente en el proceso de contacto cultural. Un claro ejemplo de este tipo de transculturación armónica se encuentra en el poema “¿Volver a África?” de Louise Bennett, que desmitifica un retorno idealizado al continente africano. Comparable con la "Balada de los dos Abuelos" de Nicolás Guillén, el poema de Bennett evoca la heterogeneidad cultural presente en la sociedad jamaicana, usando el humor como recurso crítico. Sin embargo, no diferencia entre las razones por las cuales llegaron los ancestros de África y Europa, tratándolos por iguales sin mención de la historia de la esclavitud. Un fragmento del poema traducido, al lado del original, entrega una idea de su tenor:

$\begin{array}{ll}\text { ¿Volver a África, Miss Matty? } & \text { Back to Africa, Miss Matty? } \\ \begin{array}{l}\text { ¿Sabe lo que dice usté? } \\ \text { Hay que venir de algún sitio } \\ \text { Pa' regresar luego a él. }\end{array} & \begin{array}{l}\text { Yuh noh know wha yuh dah-sey? } \\ \text { Yuh haffe come from some whe fus, } \\ \text { Before yuh go back deh? }\end{array} \\ \begin{array}{l}\text { Sé que tu tátara-tátara- } \\ \text { Tátarabuela africana fue, }\end{array} & \text { Me know sey dat yuh great great great } \\ \text { ¿pero tu tátara-tátara- } & \text { Gramma was African, } \\ \text { Tátarabuela no era inglés? } & \text { But Matty, doan yuh great great great } \\ & \text { Grampa was Englishman? } \\ \text { ¿Y el padre 'el tátara-tátara- } & \\ \text { Buelo 'e tu padre judío, jum? } & \\ \text { ¡Y tu abuelo por part'e madre } & \text { Den yuh great granmada fada } \\ \text { Fue franchute parlevú! } & \text { By yuh fada side was Jew? } \\ & \text { An yuh grampa by yuh mada side } \\ \text { Pero el resto 'e tu familia, } & \text { Was Frenchie parley-vous! } \\ \text { Los de tu generación, } & \\ \text { Todos en Bun Grung nacieron, } & \text { But de balance a yuh family, }\end{array}$

${ }^{14}$ Por "retención cultural africana" me refiero a la pervivencia de elementos africanos en las sociedades actuales del Caribe. En el área anglófona se trata de una categoría de análisis específica y ampliamente utilizada. 
Todos jamaicanos son.

$\left(\mathrm{n}^{\mathrm{o}}\right.$ 9I, II3)
Oonoo all is Jamaican!

(Bennett I04)

En general, la obra de Bennett no desatiende la esclavitud ni sus consecuencias en el racismo presente en la organización social anglocaribeña. Sin embargo, la inserción de este poema en el número lo saca de su contexto de producción y, leído solo, puede representar una armonía artificial de la identidad nacional jamaicana. Además, reproduce una generalización de los pueblos africanos mientras identifica la diferenciación de los europeos como ingleses, franceses y judíos. En términos de la traducción, Chericián no solo reproduce la rima, sino que logra recrear un lenguaje coloquial y traspasar el sentido de humor del original, ambos elementos característicos de la tradición oral cultivada por Bennett en su vasta obra poética y performática. En este sentido, el texto transfigura la estética oral para el contexto cultural cubano e hispanohablante.

Otros ejemplos de cómo la heterogeneidad cultural forma parte de la base para la construcción identitaria caribeña están presentes en "Santificado" de George Campbell y "Nosotros" de Owen Campbell, ambos traducidos por Fernández Retamar. En el primero, además de los factores africanos y europeos, evoca la importancia de los chinos e indios, que aportan a la definición de caribeños como "Tan fuertes tan originales: ¡Todos de la tierra y el sol!" (nº 9I, II 8); y, en el segundo, el hablante reclama la necesidad de quedarse en el Caribe para "Edificar aquí, en nuestro magro suelo" ( $\mathrm{n}^{-}$9I, I32). Estos mensajes también afloran en el poema "Anancy" de Andrew Salkey, traducido por Feijóo, que introduce el tradicional personaje folklórico de una araña engañadora, parte de la tradición oral africana que sobrevivió el middle passage. Aquí, sin embargo, existe una clara estrategia de recuperar y revalorizar las herencias africanas en la cultura y sociedad caribeña, como evocan los versos introductorios: "Anancy es una araña; / Anancy es un hombre; Anancy es antillano; / Anancy es africano" (n.. 91, I62). El poema pareciera manifestar que es posible ser antillano y africano a la vez, o ser un antillano-africano, al igual que lo es ser araña y hombre, como enseña la forma dual de este personaje popular.

Pese a que Casa privilegia la traducción de poesía por sobre narrativa, el balance entre los dos géneros para este dossier es bastante equilibrado. La lectura de los textos narrativos, además, está complementada por el ensayo de Benítez Rojo, “Existe una novelística antillana de lengua inglesa?", cuya función se asemeja al fundacional texto de Rama, "Diez problemas para el novelista latinoamericano", publicado en el número 26 de 1964 junto con una muestra literaria de varios autores que llegarían a integrar el boom ${ }^{15}$. Benítez Rojo ofrece un panorama de algunos de los temas

is Al igual que Rama introdujo importantes perspectivas teóricas en la crítica literaria latinoamericana, Benítez Rojo tuvo un papel fundamental en el desarrollo de la crítica caribeña en lengua española, particularmente con su noción de "la isla 
principales en la novela anglocaribeña, como la preocupación social, el viaje, el anticolonialismo y los problemas de la lengua, apoyándose en ejemplos de casi todos los novelistas publicados en el número. Un aspecto importante del ensayo de Benítez Rojo es su reflexión sobre los problemas de traducción de varios conceptos, no necesariamente abordados como tal por el autor. Así es con el barrack yard, nombre de las típicas viviendas sociales del Caribe anglófono, que aparece en la narrativa de los trinitarios Alfred Mendes y C.L.R. James desde los años 3o. Si bien no identifica el subgénero narrativo del barrack yard ${ }^{16}$, asemeja este espacio social con el solar cubano y destaca que los autores lo evocan "Para revelar un mundo sórdido y de inconformidad social" (n.o 9I, I9I). El término que quizás genera el mayor problema de traducción es el de creol, probablemente por ser un fenómeno lingüístico poco familiar en español. Siguiendo la crítica anglocaribeña del momento, Benítez Rojo prefiere hablar de "dialecto" en vez de "creol", lo que genera una confusión conceptual: señala que, "El llamado dialecto, inglés ingenuo y maltrecho que hablan los personajes del pueblo, nace del primitivo creole, el único idioma que pudo unir a los esclavos de distintas procedencias en torno a algún desesperado proyecto de evasión o de rebelión en el pasado" (cursivas en el original, n⿳o 9I, I88). Si bien describe el creol como un idioma, la definición que aporta corresponde más a un pidgin, es decir, la primera manifestación de un nuevo idioma producto del contacto cultural entre dos o más lenguas ${ }^{17}$ (McWhorter ${ }_{4-5}$ ). No obstante, Benítez Rojo reconoce que el dialecto es "la característica más importante de esta literatura, con aquella que la individualiza con mayor autenticidad" (ก.ำ 9I, I88). De los autores que logran utilizar mejor el llamado dialecto en su narrativa, destaca a Wilson Harris, comparando su novela Palace of the peacock (1960) con Pedro Páramo (1955) y Los pasos perdidos (1953). Concluye que, "más que una traba, [el dialecto] representa un ventajoso punto de partida, nítidamente diferenciado respecto a otras literaturas, en las cuales las posibilidades de recrear un lenguaje comunicable son sin dudas más limitadas" (n.o 9I, r9o). Estas discrepancias revelan la incapacidad de traducir al contexto cubano uno de los fenómenos lingüísticos más definitorios de la cultura popular en el Caribe anglófono, problematizando el

que se repite", metáfora que destaca las similitudes en las diversas manifestaciones culturales del Caribe a partir de las sociedades de plantación. Para mayor profundidad, véase su libro La isla que se repite: El Caribe y la perspectiva postmoderna (1989). Es interesante que, según Rafael Rojas, el archivo antillano que utiliza Benítez Rojo en este libro corresponde principalmente a un caribeñismo cubano, basado en el trabajo de Fernando Ortiz, Manuel Moreno Fraginals y José Luciano Franco, y no tanto en la producción intelectual de las otras zonas lingüísticas de la región, aunque reconoce la presencia de referentes como Aimé Césaire, Eric Williams, Franklin Knight y Wilson Harris (168-i69). Con todo, Benítez Rojo contribuyó de manera singular a la difusión de la producción cultural del Caribe no hispanohablante en Cuba y América Latina, como escritor, crítico y el primer director del Centro de Estudios del Caribe, dependiente de la Casa de las Américas.

${ }^{16}$ Para una mayor elaboración sobre este subgénero, véase: Reinhard Sander. The Trinidad Awakening. West Indian Literature of the Nineteen-Thirties. Nueva York: Greenwood Press, 1988, en particular el capitulo 3. En Chile, este espacio se asemeja más al conventillo.

${ }^{17}$ McWhorter plantea que todos los creoles caribeños pasaron por un proceso de pidginización que puede haber ocurrido en las plantaciones del Caribe y/o en la costa occidental de África (4-5). 
discurso nuestroamericano promovido por la revista y explicitado con frecuencia en las colaboraciones sobre la producción cultural caribeña de otras expresiones lingüisticas. El hecho de que el poema de Bennett incluido en el dossier es el único texto escrito en una forma de creol, también sugiere las distancias culturales entre estas dos zonas lingüísticas del Caribe.

\section{CODA PARA UN PROYECTO PANCARIBEÑO}

En la segunda mitad de los 7o, los intercambios entre Cuba y el Caribe aumentaron considerablemente, facilitados en gran parte por las buenas relaciones diplomáticas entre el gobierno de Fidel Castro y los de Michael Manley (Jamaica), Forbes Burnham (Guyana) y Eric Williams (Trinidad y Tobago). En el ámbito cultural, el universo caribeño se curvó y se cruzaron los estallidos casi paralelos de la literatura anglocaribeña y latinoamericana, para usar la metáfora de Lucía Stecher ("Dos estallidos..." I26). Así lo comprueba una serie de cambios estructurales e hitos en la Casa que ayudan a comprender el desarrollo de sus políticas culturales en esta época: en 1976, el Premio Literario incorpora la literatura caribeña escrita en inglés, seguido tres años después por la incorporación de la literatura caribeña escrita en francés; el mismo año I979, la Casa crea el Centro de Estudios del Caribe y en 198I se funda su propia revista, Anales del Caribe, que publica - hasta hoy - trabajos originales en las principales lenguas de la región, incluyendo los variantes de creol. Finalmente, en 1983, el Premio Literario precisa su definición de literaria caribeña no hispanohablante, incorporando la admisión de obras escritas en creol, tanto en sus variantes anglófonas como francófonas. Estos hitos, para Fernández Retamar, significaron "la plena inserción del Caribe en el horizonte de la Casa de las Américas" ("La Casa..." párr. 9). Al entrar a la década de los 8o, las relaciones entre Cuba y algunos países del Caribe anglófono se vieron fuertemente afectadas, disminuyendo el flujo de intercambio entre la Casa y la intelectualidad anglocaribeña. En Jamaica, por ejemplo, las elecciones de ig8o dieron el poder a Edward Seaga, del conservador Jamaica Labour Party, quien cortó relaciones diplomáticas con el gobierno de Castro, dando fin a una década de colaboración política y cultural entre las dos islas. En este contexto, la continuación de la categoría de literatura caribeña escrita en inglés o creol en el Premio Literario demuestra la perseverancia del proyecto pancaribeño de la Casa que cautivó a varios escritores de la región, como Kamau Brathwaite y George Lamming, y también a Andrew Salkey y Jan Carew.

Pese a la colaboración constante de estos autores, los intercambios entre la intelectualidad cubana y anglocaribeña no volverían a tener el mismo tipo de efervescencia alcanzada en los años 7o, un momento que, pese al tenso ambiente cultural en Cuba, significó una de las mayores 
aperturas hacia otra zona lingüística en la historia de la revista e institución. Estos intercambios generalmente han sido leídos como una maniobra política en el contexto del creciente aislamiento de Cuba por parte de los gobiernos latinoamericanos y una facción de escritores latinoamericanos. Mientras estos factores pueden haber impulsado la búsqueda de aliados políticos dentro de la región, la rearticulación del discurso nuestroamericano en la revista Casa pone en vista la evolución de un proyecto editorial comprometido con la creación de una comunidad de escritores e intelectuales de todas las regiones de la América subdesarrollada. Por otra parte, la novedad de autorías del Caribe anglófono que introduce la revista en esta época puede haber contribuido a la desintegración de cierta sensación de intolerancia hacia el final del llamado quinquenio gris, aunque, como publicación hegemónica en el campo literario cubano, los autores anglocaribeños también pueden haber sido asociados a las políticas culturales oficiales. Como sea, la preocupación por la identidad cultural caribeña que manifiestan los colabores de Jamaica, Barbados, Guyana y Trinidad y Tobago, enfatiza no solo perspectivas anticoloniales, sino también de reivindicación racial, que, si bien es afín al proyecto político de la Casa, encuentra puntos disonantes en cuanto al discurso de la transculturación armónica en Cuba.

Al transformar la traducción en el objeto de análisis de la revista, emerge la anatomía del discurso nuestroamericano y el alcance de sus retóricas en el campo cultural cubano y latinoamericano. Los criterios de selección de autores, la traducción o no traducción de conceptos, las estrategias de validación de obras, la visibilidad de los traductores y las lenguas desde los cuales se traduce, las reflexiones sobre el proceso de traducción dibujan un entramado complejo que permite comprobar que Casa, al menos durante sus primeros treinta años, considera la traducción como una práctica literaria e intelectual legítima y necesaria para su proyección continental. Este espacio de intercambio virtual y presencial que la revista construye con la producción literaria del Caribe anglófono revela las coincidencias y disonancias propias de cualquier espacio de inteligibilidad entre dos o más universos culturales. Pero también significa un caso anómalo en la historia cultural del Caribe y un punto de inflexión para los futuros esfuerzos pancaribeños.

\section{RECONOCIMIENTOS}

Este artículo forma parte del proyecto "Connected Worlds: The Caribbean, Origin of Modern World" y ha recibido financiamiento del programa de investigación e innovación Horizon 2020, bajo el acuerdo de beca de Marie Sklodowska Curie, de la Unión Europea, por el № 823846 / This article forms part of the project "Connected Worlds: The Caribbean, Origin of Modern World" 
and has has received funding from the European Union's Horizon 2020 research and innovation programme under the Marie Sklodowska Curie grant agreement № 823846 .

\section{REFERENCIAS}

Acosta, Blanca, Samuel Goldberg and Ileana Sanz. Caribbean Stories. Barbados, Guyana, Jamaica, Trinidad-Tobago. La Habana: Casa de las Américas, 1977

Bennett, Louise. Selected Poems. Mervyn Morris, ed. Kingston: Sangster's Book Stores Ltd., 2005.

Benítez Rojo, Antonio. La isla que se repite. El Caribe y la perspectiva postmoderna. Hanover: Ediciones del Norte, 1989 .

Casa de las Américas. La Habana: Casa de las Américas, r960-presente.

Campuzano, Luisa. "La revista Casa de las Américas, 1960-1995". La revista Casa de las Américas: un proyecto continental. Fornet y Campuzano. La Habana: Centro de Investigación y Desarrollo de la Cultura Cubana, (200I): 3I-70.

Caribbean Quarterly. Mona, Kingston: University of the West Indies, 1945-presente.

Ellis, Keith, sel., intro. y notas. Poetas del Caribe anglófono. Tomo r. La Habana: Casa de las Américas, 2OII.

Fernández Retamar, Roberto. Carta a Andrew Salkey. 15 julio 1974. Expediente de Andrew Salkey, Departamento de Memoria, Archivo de Casa de las Américas, La Habana.

Fernández Retamar, Roberto. "El Caribe en la revista Casa de las Américas". Anales del Caribe (2016): I78-I85.

Fernández Retamar, Roberto. "La Casa de las Américas y el Caribe". La Jiribilla. Año IX, I6-22 abril, 20II. Visitado el iz de marzo de 2020. https://cutt.ly/umbHqtx

Fernández Retamar, Roberto. Un poeta metido en camisa de once varas. La Habana: Ediciones UNIÓN, 2018.

Fornet, Ambosio. "Casa de las Américas: entre la Revolución y la Utopía". La revista Casa de las Américas: un proyecto continental. Fornet y Campuzano. La Habana: Centro de Investigación y Desarrollo de la Cultura Cubana, (200I): 7-29.

Freyre, Gilberto. Casa-Grande y Senzala. Trad. Benjamín de Garay y Lucrecia Manduca. Caracas: Biblioteca Ayacucho. 1985.

Gilman, Claudia. Entre la pluma y el fusil. Debates y dilemas del escritor revolucionario en América Latina. Buenos Aires: Siglo Veintiuno Editores, 2003. 
Gilman, Claudia. "Los primeros años de Casa de las Américas: estética política en la construcción de una red" Escrituras en tránsito. Revistas y redes culturales en América Latina. Ed. César Zamorano Díaz. Santiago: Editorial Cuarto Propio, (2018): I29-153.

Guzmán, María Constanza "El Caribe se traduce: la traducción como praxis descolonial en las revistas Tropiques, Bim y Casa de las Américas". Mutatis Mutandis, I0.I (2017): I67-18I.

Jakobson, Roman. "On Linguistic Aspects of Translation". The Translation Studies Reader. $3^{\underline{a}}$ ed. Lawrence Venuti, Ed. Londres: Routledge, г26-гзг.

Lie, Nadia. Transición y transacción. La revista cubana Casa de las Américas (1960-1976). Gaithersburg, MD, EEUU: Hispamérica, 1996.

McWhorter, John H. The Missing Spanish Creoles. Recovering the Birth of Plantation Contact Languages. Berkeley: University of California Press, 2000.

Morejón Arnaiz, Idalia. Política y polémica en América Latina. Las revistas Casa de las Américas y Mundo Nuevo. Leiden, Países Bajos: Almenara, 2017.

Rodríguez, Carlos Rafael. "Fundamentos estratégicos de la política exterior de Cuba". Casa de las Américas I3I (1982): II-2I.

Rodríguez-Carranza, Luz y Nadia Lie. "A Comparative Analysis of Caribbean Literary Magazines: 1960-1980". A History of Literature in the Caribbean (Vol. 3). Arnold, ed. Amsterdam: John Benjamins, (1997): I19-160.

Rojas, Rafael. Viajes del saber. Ensayos sobre lectura y traducción en Cuba. Leiden, Países Bajos: Almenara, 2018.

Sanz, Ileana. "La complejidad lingüística del Caribe: desafíos para un traductor literarios" Anales del Caribe (2018): 175-185.

Stecher, Lucía. "Dos estallidos paralelos y un universo que se curva: encuentros y desencuentros entre el Caribe e Hispanoamérica". Casa de las Américas 277 (2014): I20-Izo.

Walcott, Derek. Collected Poems 1948-1984. London: Faber and Faber, 1992.

Weiss, Judith A. Casa de las Américas: An Intellectual Review in the Cuban Revolution. Tesis de Doctorado. Yale University, I973. PDF.

Willson, Patricia. La constelación del sur: traductores y traducciones en la literatura argentina del siglo $X X$. Buenos Aires: Siglo Veintiuno Editores, 2017. 